\title{
INFLUENCE OF MAMMOGRAPHY SCREENING IN THE TREATMENT OF WOMEN DIAGNOSED WITH BREAST CANCER
}

\author{
Luiza da Rosa Ramos', Laura Becker Carminatti², Laura Gazola Ugioni² \\ ${ }^{1}$ Hospital São José - Criciúma (SC), Brazil. \\ ¿Universidade do Extremo Sul Catarinense - Criciúma (SC), Brazil.
}

Introduction: Apart from non-melanoma skin tumors, breast cancer is the most prevalent neoplasm among women in Brazil and worldwide. Breast cancer is a very heterogeneous disease, a fact attributed to the plasticity of its cells. Therefore, the stratification of tumors is critical to achieving better clinical outcomes. Breast cancer screening often allows diagnosing the disease in its earlier stages, manifested as smaller tumors, and still without lymph node involvement. The multiple prognostic factors that must be taken into account when considering the eligibility for treatment, such as age, reproductive status, type of cancer, and severity of the disease, make it impossible to establish clear approach guidelines for the disease, given the many different clinical situations. Objectives: To evaluate the influence of mammography screening in the treatment of women with a previous diagnosis of breast cancer. Methodology: This is an observational, descriptive, cross-sectional study conducted with primary and secondary data and a quantitative approach. The research was carried out in a high-complexity hospital in the far-south of Santa Catarina, Southern Brazil, and assessed patients with a previous diagnosis of breast cancer, from 2012 to 2017, who were on oncological outpatient follow-up. Results: Among the 99 patients analyzed, 58.6\% undergo the examination annually, and $49.5 \%$ were diagnosed less than 12 months after the last mammography. Stage I diseases were more frequent, corroborating the finding that $74.7 \%$ of patients were submitted to conservative surgeries and $68.7 \%$ to sentinel lymph node biopsy, instead of more extensive procedures. With respect to the treatment of choice, patients with annual or biennial mammography frequency had surgical and chemotherapeutic outcomes similar to those of women who had no set frequency or who had never had a screening, that is, $72.4 \%$ of patients with annual frequency and $100 \%$ of patients with biennial frequency were submitted to conservative surgery, as occurred with $85 \%$ of patients with no set frequency and $66 \%$ of those who had never had the test. Conclusion: Patients who had annual mammographies and those diagnosed less than 12 months after the last mammography presented smaller tumors at diagnosis, but these characteristics did not influence the type of treatment chosen. 